\title{
Performance of horn-coupled transition edge sensors for $L$ - and S-band optical detection on the SAFARI instrument
}

\author{
D. J. Goldie ${ }^{a,}$, D. M. Glowacka ${ }^{a}$, S. Withington ${ }^{a}$, Jiajun Chen ${ }^{a}$, P. A. R. Ade ${ }^{b}$, D. Morozov ${ }^{b}$, R.

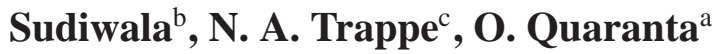 \\ ${ }^{a}$ Quantum Sensors Group, Cavendish Laboratory, University of Cambridge, JJ Thomson Av., Cambridge, UK, CB3 \\ OHE \\ ${ }^{\mathrm{b}}$ School of Physics and Astronomy, Cardiff University, Cardiff, UK, CF24 3 YB \\ ${ }^{\mathrm{c}}$ Department of Experimental Physics, National University of Ireland, Maynooth, Ireland
}

\begin{abstract}
We describe the geometry, architecture, dark- and optical performance of ultra-low-noise transition edge sensors as THz detectors for the SAFARI instrument. The TESs are fabricated from superconducting Mo/Au bilayers coupled to impedance-matched superconducting $\beta$-phase Ta thin-film absorbers. The detectors have phonon-limited dark noise equivalent powers of order $0.5-1.0 \mathrm{aW} / \sqrt{\mathrm{Hz}}$ and saturation powers of order $20-40 \mathrm{fW}$. The low temperature test configuration incorporating micro-machined backshorts is also described, and construction and typical performance characteristics for the optical load are shown. We report preliminary measurements of the optical performance of these TESs for two SAFARI bands; L-band at $110-210 \mu \mathrm{m}$ and S-band $34-60 \mu \mathrm{m}$.
\end{abstract}

Keywords: Transition edge sensor, far-infrared bolometer, optical characterization, ultra-low noise.

*D. J. Goldie, d.j.goldie@mrao.cam.ac.uk

\section{Introduction}

The proposed space instrument SAFARI is a far-infrared imaging Fourier spectrometer that will operate at Lagrange point two with a $3.2 \mathrm{~m}$ primary mirror cooled to $6 \mathrm{~K} .^{1,2}$ The instrument comprises three detector arrays to perform imaging spectroscopy in three bands: $34-60 \mu \mathrm{m}$ (S-band), $60-110 \mu \mathrm{m}$ (M-Band) and 110-320 $\mu \mathrm{m}$ (L-band). The low mirror temperature enables backgroundlimited astronomical observation but also demands optically-coupled detectors of outstanding performance. At the heart of these detectors will be transition edge sensors (TESs) operating at a temperature close to $100 \mathrm{mK}$ and the TESs must be coupled to the incident optical power. Target dark noise equivalent powers for the instrument are $0.2 \mathrm{aW} / \sqrt{ } \mathrm{Hz}$ for $\mathrm{M}$ - and L-band and $0.25 \mathrm{aW} / \sqrt{ } \mathrm{Hz}$ for S-band pixels. Previously we have reported dark and optical measurements of TES L-band pixels with pyramidal horns and mechanically machined hemispherical backshorts. ${ }^{3}$ Audley et al. ${ }^{4}$ and Khoshropanah ${ }^{5}$ have reported dark and optical measurements of S-band pixels with pyramidal horns in a similar configuration.

The goal of the current programme was to significantly enhance the technology readiness level of this earlier work. Amongst our aims were:

- To configure a precision optical test facility to characterize ultra-low-noise THz detectors,

- In an 'optically clean’ configuration with known throughput,

- With minimal optical stray-light contamination,

- And to quantify the optical performance of horn-coupled, ultra-low-noise TESs at L- and Sband.

Millimeter, Submillimeter, and Far-Infrared Detectors and Instrumentation for Astronomy VIII, edited by Wayne S. Holland, Jonas Zmuidzinas, Proc. of SPIE Vol. 9914, 99140A

(C) 2016 SPIE · CCC code: 0277-786X/16/\$18 · doi: 10.1117/12.2232740

Proc. of SPIE Vol. $991499140 \mathrm{~A}-1$ 
- To demonstrate and quantify the assembly and integration procedures required to realize the large sensor and horn arrays required by the SAFARI instrument,

- To realize detectors with identical optical configurations for both ultra-low and very-high power loadings; the latter for spatial and spectral beam-pattern characterization with roomtemperature sources.

- The development, demonstration and characterization of a precision micro-machined backshort technology, capable of immediate extrapolation to the large arrays required by SAFARI at all wavelengths.

- To realize and characterize an arrayable technology for large arrays of profiled, pyramidal, optical horns at all SAFARI wavelengths.

In this paper it is not possible to report the progress in all aspects of these areas. However, in Sec. 2 we describe the fabrication of L- and S-band detectors and the micro-machined flat backshorts. In Sec. 3 we describe the optical test system. Section 4 briefly describes the fabrication of the profiled pyramidal horns used in this work and Sec. 5 discusses the integration of the detectors into the optical test system. Section 6 describes how detected power was quantified, gives results of dark and optical measurements, and also shows results of measurements of inter-pixel crosstalk and stray light. An accompanying paper gives details of the horn manufacture, and the spatial and spectral measurements of the system response using high-power handling TESs. ${ }^{6}$

\section{Prototype pixels}

The detectors described here are thin-film Mo-Au bilayer transition edge sensors (TESs) with superconducting $\beta$-phase Ta absorbers. They are thermally isolated from a low-temperature $65 \mathrm{mK}$ refrigeration stage by long, thin silicon nitride $\left(\mathrm{SiN}_{\mathrm{x}}\right)$ support structures in order to achieve the low thermal conductance needed to reach the NEP requirement. The detectors are coupled to flat backshorts to maximize optical coupling.

\subsection{TES Detectors}

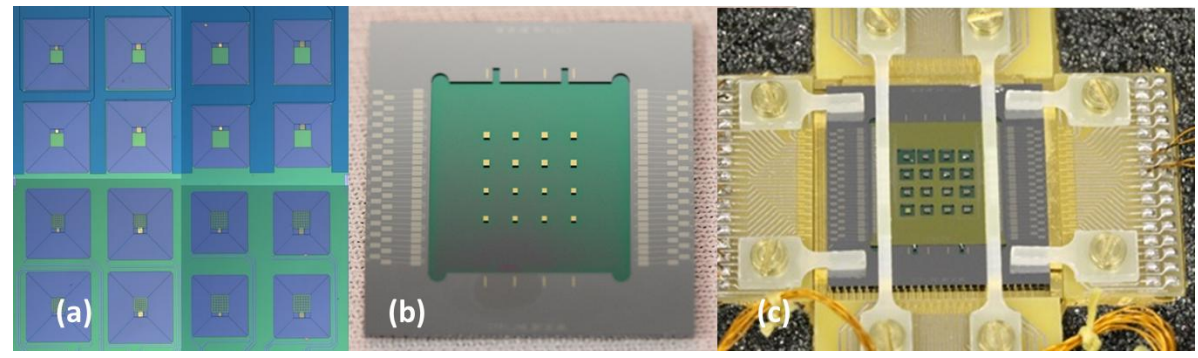

Fig 1: Images of the detector chip and assembly: (a) Composite image of an L-band $4 \times 4$ prototype array. The upper two rows show filled absorbers the lower 2 rows are meshed. (b) Backing plate prior to assembly and, (c) the array mounted on the backing plate in the test system. 
These prototype detectors were fabricated as $4 \times 4$ arrays on $50 \mathrm{~mm}$-diameter Si wafers coated by PECVD with $200 \mathrm{~nm}$ amorphous silicon nitride $\left(\mathrm{SiN}_{\mathrm{x}}\right)$. The TESs were fabricated from $\mathrm{Mo} / \mathrm{Au}$ bilayers with thicknesses 40/150 nm for the L- and 40/170 nm for S-band detectors giving superconducting transition temperatures $\left(T_{c}\right)$ of $180-200$ and $130-140 \mathrm{mk}$ for the two bands respectively. The transition temperatures were chosen to enhance detector saturation powers in the event, for example, of stray-light in the measurement system rather than optimizing detector sensitivity, minimizing the noise equivalent power. The TESs were closely thermally coupled to square, thin-film $\beta$-phase Ta optical absorbers with $T_{c} \simeq 870 \mathrm{mK}$ having dimensions 320 or $250 \mu \mathrm{m}$ for L- and 160 or $78 \mu \mathrm{m}$ for S-band. Both filled and meshed absorber geometries were present. The filling factor for the meshed absorbers was close to $50 \%$. The normal-state Ta resistance was $R_{s q}=440 \Omega$ closely matching the filled absorbers to free-space impedance $\left(Z_{0}=388 \Omega\right)$, although somewhat mismatching the meshed absorbers of filling-factor $\sim 50 \%$. The filled and meshed absorbers were formed from the same Ta deposition. Electrical contacts to the TES were formed from superconducting $\mathrm{Nb}$ using both planar and microstrip wiring configurations. The $\mathrm{SiN}_{\mathrm{x}}$ was patterned by reactive ion etching (RIE) and the Si wafer itself was etched by deep RIE (DRIE). Details of the fabrication can be found in Ref. 7.

\subsection{Backing plates}

The backing plates consisted of optically-flat backshorts that were coated with high-conductivity sputtered $\mathrm{Au}$, a recess within which the array was precisely positioned and superconducting fan-out wiring. Backing plates were fabricated on $100 \mathrm{~mm}$-diameter silicon-on-insulator (SoI) wafers. The SoI device-layer thickness was chosen to ensure that the backshorts were positioned $\lambda_{c} / 4$ behind the Ta absorbers, $\lambda_{c}$ is the band-centre wavelength. The device-layer was etched to form the pillars of the backshorts and, at the same time, the recess within which the detector chip was positioned. Superconducting wire bonds connected the device wiring to $\mathrm{Nb}$ fan-out wiring on the backing plate and then to the bias circuit and SQUID readout. Figure 1 shows optical images of (a) a prototype $4 \times 4$ array L-band array, (b) an L-band backing plate before assembly and, (c) the assembled array and backing plate in the test system.

\section{The optical test system}

The optical test system uses an adiabatic diamagnetic refrigerator (ADR) attached to a pulse tube cooler (PTC). The PTC operates at $3.3 \mathrm{~K}$ and the two-stage ADR gives an intermediate platform at $1 \mathrm{~K}$ and a cold stage with bath temperature $\left(T_{\text {bath }}\right)$ of $65 \mathrm{mK}$. A cryogenic blackbody load was designed to allow optical illumination of the devices. The blackbody included thermal-blocking and band-defining optical filters, and an optical aperture. Figure 2 shows an engineering drawing of the optical test system. The bandpass filters and aperture were mounted on the cold-stage, and these filters were angled to prevent multiple reflections. A thermal-blocking filter was mounted on the $3 \mathrm{~K}$ stage to minimize long-wavelength loading of the bandpass filters. This clean optical path ensures that the optical characteristics can be precisely calculated. The hot-load was mechanically anchored to the $3 \mathrm{~K}$ stage by a thermally-isolating Kevlar suspension, and thermally connected by a $\mathrm{Cu}$ wire allowing optimization of both the thermal loading onto the $3 \mathrm{~K}$ stage and the load response time. The load was thermally isolated from the cold stage by an optically-blackened labyrinth, highlighted in the inset of the figure. In addition, all internal components of the load were optically-blackened to minimize any reflections. Readout electronics used superconducting quantum interference devices 


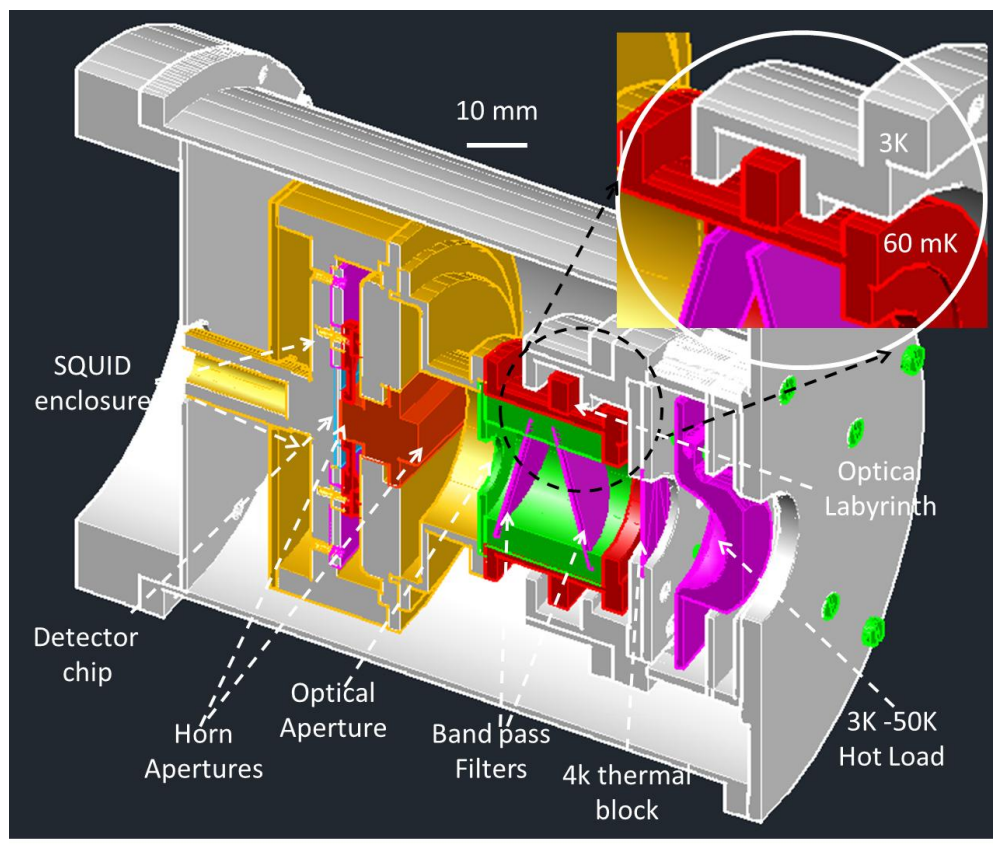

Fig 2: Engineering drawing of the camera construction. The detectors, horns, optical aperture and band-defining filters are thermally isolated from the outer $3 \mathrm{~K}$ shield and hot load by an opticallyblackened labyrinth shown in the inset (upper right). The hot-load (shown in purple to the right of the schematic) was loosely thermally-coupled to the $3 \mathrm{~K}$ stage.

(SQUIDS) with voltage bias of the TESs. The readout was positioned in a separate enclosure behind the detectors (indicated in the left of the figure) and electrically connected via a light-tight feedthrough into the detector enclosure. A PID feedback system was implemented to minimize the effect of thermal loading from the hot load onto the cold stage. For the optical tests $T_{\text {bath }}$ was regulated to $90 \mathrm{mK}$ to maximize available measurement time, detector sensitivity and dynamic range.

\section{Horn Arrays}

The feed-horns for both L- and S-bands were square, profiled pyramidal horns and were fabricated using wire erosion of an aluminium block forming a mandrel. Copper was electro-formed onto the mandrel and the Al was removed by chemical etching. The horn arrays were completed by $\mathrm{Au}$ plating, precision machining and polishing. The horns were manufactured by Thomas Keating Ltd. ${ }^{8}$ Images of the entrance apertures of a prototype S-band array can be seen in the Fig 3 (b). For Lband horns the square entrance apertures are $1450 \mu \mathrm{m}$ wide at $1600 \mu \mathrm{m}$ centers with exit apertures of $235 \mu \mathrm{m}$. For the S-band horns entrance apertures are $662 \mu \mathrm{m}$ wide at $812 \mu \mathrm{m}$ centers with exit apertures of $68 \mu \mathrm{m}$. Further details of the horn manufacture and performance are given in Ref. 6 .

\section{Assembly and Integration}

Figure 4 shows an example of the metrology used during integration of the backing plates, detectors and horns. Figure 4a shows an image of an S-band array mounted on a backing plate and the dashed line indicates the line of the measurement. Figure 4 shows the surface measurement obtained with a 


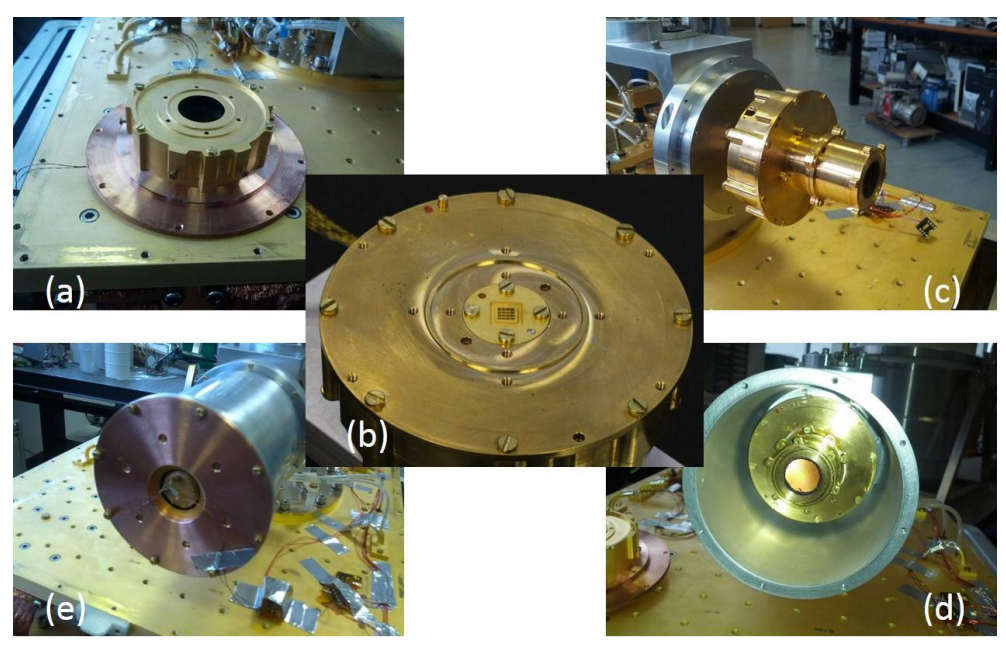

Fig 3: Photographs of the blackbody assembly: (a) The detector housing. For dark testing the central aperture can be blanked. (b) The horn plate mounted-the entrance apertures of an S-band horn array can be seen in the centre of the image. (c) With the filter stack attached. (d) View of the outer-most band-defining filter from the aspect of the blackbody, and (e) the complete camera with primary $3 \mathrm{~K}$ thermal shield in place before installation of the outer $3 \mathrm{~K}$ thermal and magnetic shielding.

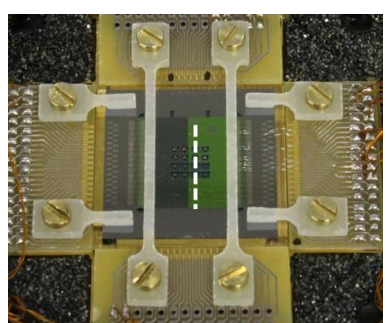

(a)

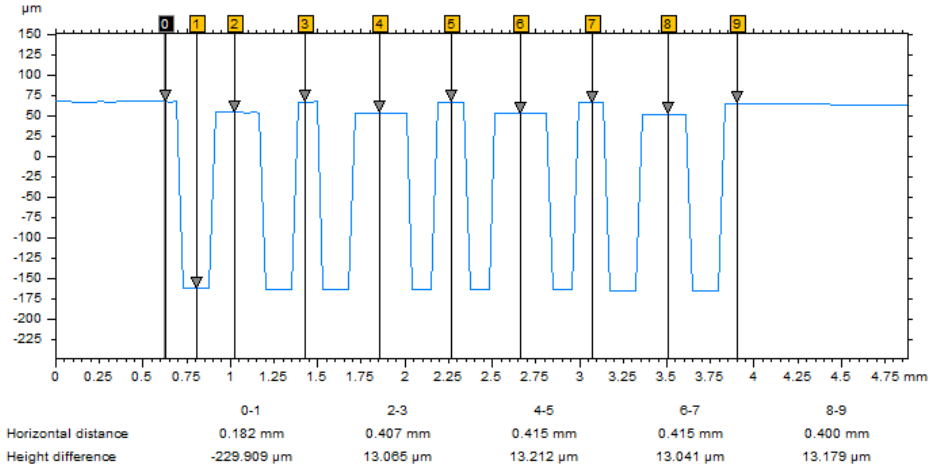

(b)

Fig 4: (a) Image of an S-band chip and (white-dashed line) the line of the measurement. (b) Height differences: the Au reflector on the backing plate is $13.2 \pm 0.1 \mu \mathrm{m}$ behind the absorbers.

non-contact Polytech surface profiler. ${ }^{9}$ Steps from the detector surface (arrows 0, 3, 5, 7 and 9) onto the four backshort surfaces (arrows 2, 4, 6 and 8) are visible. Height differences are $13.2 \pm 0.1 \mu \mathrm{m}$. This emphasizes the precision that can be achieved using the micro-machined SoI flat backshort. A similar non-contact measurement allowed precise positioning of the horn exit apertures at $20 \pm 1 \mu \mathrm{m}$ in front of the absorber surfaces. 


\section{Measurement and Results}

\subsection{Dark Measurements}

Dark measurements reported here were performed on 6 pixels from both L-band and S-bands. The L-band pixels had $T_{c}$ in the range $180-200 \mathrm{mK}$. Bath conductances and saturation powers were in the range $G_{b} \sim 300-700 \mathrm{fW} / \mathrm{K}, P_{\text {sat }} \sim 30-60 \mathrm{fW}$ dependent on leg length. Phonon-limited dark NEP's were in the range $0.65-1 \mathrm{aW} / \sqrt{\mathrm{Hz}}$.

S-band pixels had $T_{c}$ in the range $130-140 \mathrm{mK}, G_{b} \sim 500-900 \mathrm{fW} / \mathrm{K}$ and $P_{\text {sat }} \sim 20-50 \mathrm{fW}$. Phonon-limited dark NEP's were in the range $0.5-0.75 \mathrm{aW} / \sqrt{\mathrm{Hz}}$.

\subsection{Optical Measurements}
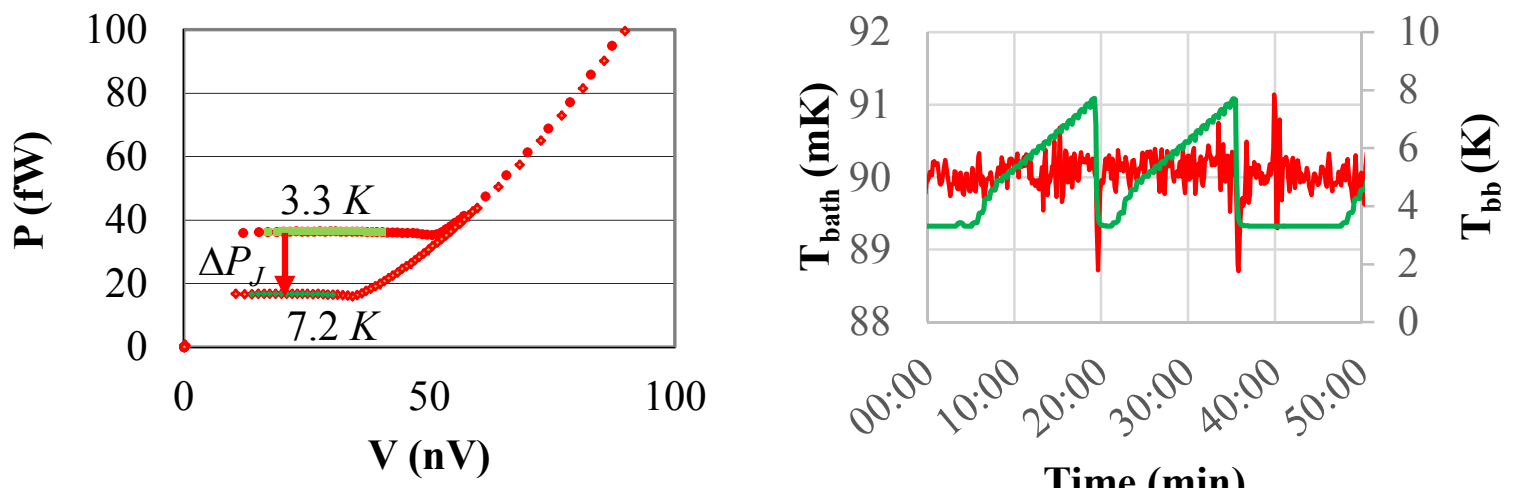

Time (min)

Fig 5: Measurement of the detected power. (a) Detected power was determined from the change in TES Joule power as the blackbody temperature was increased from $3.3 \mathrm{~K}$ the blackbody base temperature. (b) The bath temperature (redline) was regulated at $90 \mathrm{mK}$ as $T_{b b}$ (green line) was changed.

The detected optical power $\Delta P_{J}$ as a function of blackbody load temperature $T_{b b}$ was determined from measured TES current-voltage characteristics from which TES Joule bias power $P_{J}\left(T_{b b}\right)$ was determined. $\Delta P_{J}$ is given by

$$
\Delta P_{J}=P_{J}(0)-P_{J}\left(T_{b b}\right) .
$$

With $P_{J}(0)$ the TES Joule power with $T_{b b}=3.3 \mathrm{~K}$, the base temperature of the blackbody load. The detected power is compared to the change in incident optical power of a single mode with two polarizations

$$
\Delta P_{o p t}=P_{o p t}\left(T_{b b}\right)-P_{o p t}(0),
$$

and as previously $P_{\text {opt }}(0)=P_{\text {opt }}(3.3 \mathrm{~K})$ the incident optical power at the load base temperature. The incident optical power at $T_{b b}$ for a single mode with two polarizations is

$$
P_{\text {opt }}\left(T_{b b}\right)=\int_{\lambda_{\min }}^{\lambda_{\max }} \frac{2 h c^{2}}{\lambda^{3}} f\left(\lambda, T_{b b}\right) \tau(\lambda) d \lambda,
$$


where the Planck function $f\left(\lambda, T_{b b}\right)=\left(\exp \left(h c / \lambda k_{b} T_{b b}\right)-1\right)^{-1}, h$ is Planck's constant, $k_{b}$ is Boltzmann's constant and $c$ is the speed of light. $\tau(\lambda)$ is the measured filter response, and the filter response and waveguide size determine $\lambda_{\min }$ and $\lambda_{\max }$. We then quantify the measurements in terms of an effective number of detected modes

$$
\eta_{e f f}=\Delta P_{J} / \Delta P_{o p t}
$$

An example of the change of the Joule bias of a TES as $T_{b b}$ was changed is shown in Fig 5 (a). Fig 5 (b) shows the regulation of $T_{b a t h}$ at $90 \mathrm{mK}$ with changing $T_{b b}$ as a series of these measurements were made. $T_{\text {bath }}$ was constant to $\pm 200 \mu \mathrm{K}$ for all of the optical measurements.

\subsubsection{L-band measurements}

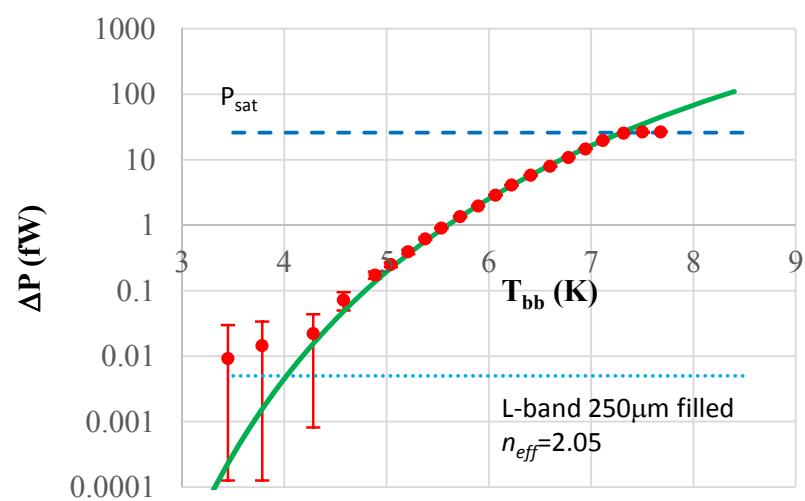

Fig 6: Detected power (red circles) as a function of $T_{b b}$ for an L-band pixel. The green line is the calculated power predicted by Eq. 3 scaled by an effective number of modes $\eta_{\text {eff }}=2.05$.

Figure 6 shows the detected optical power for an L-band sensor with Ta-absorber film of measured sheet resistance $R_{s q}=440 \Omega$ closely matched to free-space impedance $\left(Z_{0}=388 \Omega\right.$ ). The absorbing Ta film was a filled square absorber of side length $250 \mu \mathrm{m}$. The red circles show the detected power as a function of $T_{b b}$. The green solid line is the calculated power from Eq. 3 multiplied by an effective number of detected modes $\eta=2.05$ in this instance. Evidently the pyramidal horns behave in a few-moded fashion as expected. The upper dashed blue line indicates the saturation power of the TES. The lower dotted blue line indicates the system noise floor for the measurements - this was slightly higher than the expected noise calculated from the phonon-limited NEP and the measurement bandwidth in these experiments. Even so the measurement dynamic range exceeds $37 \mathrm{~dB}$.

We found $\eta_{e f f}=1.95 \pm 0.4$ for 4 filled L-band pixels and $\eta_{e f f}=1.24 \pm 0.05$ for 2 meshed Lband pixels, independent of absorber size $(320$ or $250 \mu \mathrm{m})$. The reduction in $\eta_{\text {eff }}$ between the filled and meshed absorbers was as expected based on the impedance mismatch of the meshed absorbers to $Z_{0}$ and of order $40 \%$.

\subsubsection{S-band measurements}

Figure 7 shows the detected optical power for an S-band pixel with a filled absorbing Ta film of side length $160 \mu \mathrm{m}$ and sheet resistance $R_{s q}=440 \Omega$. The red circles show the detected power as 


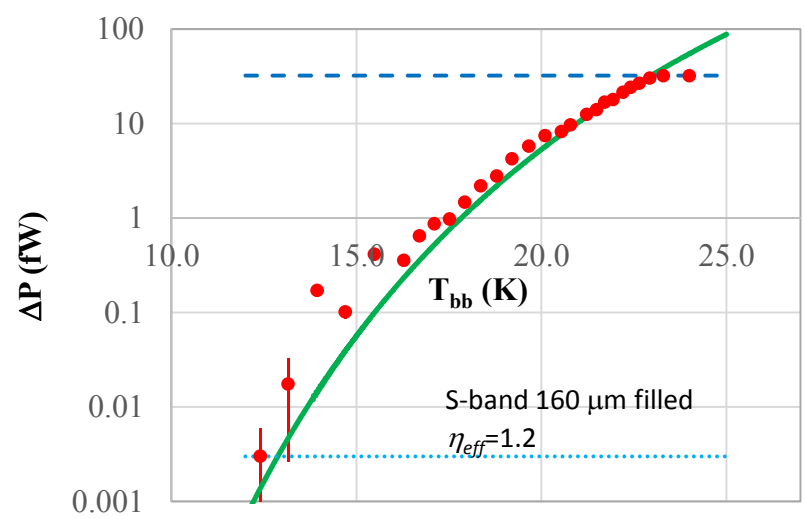

Fig 7: Detected power (red circles) as a function of $T_{b b}$ for an S-band pixel. The green line is the calculated power predicted by Eq. 3 scaled by an effective number of modes $\eta_{\text {eff }}=1.2$.

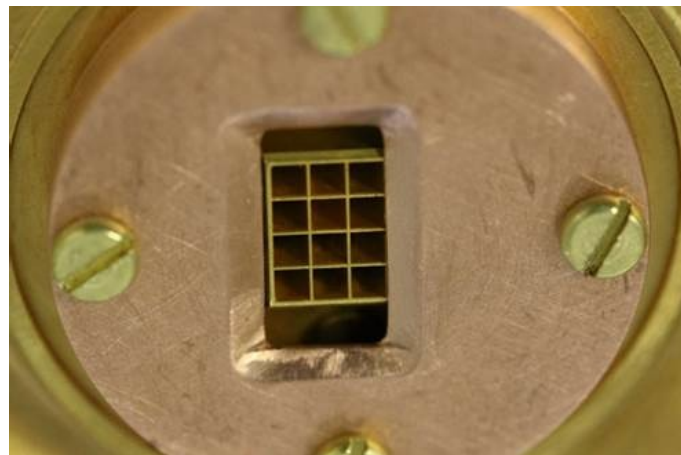

(a)

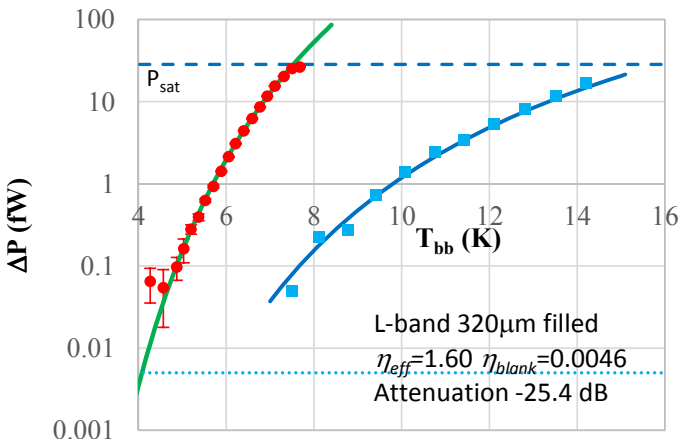

(b)

Fig 8: The (red) circles show the detected power as a function of $T_{b b}$. The green line is the calculated power with an effective number of modes $\eta_{\text {eff }}=1.6$. The (blue) squares show the detected power for the same pixel when the horn entrance is blanked. The power is characterized as an effective number of modes $\eta_{\text {blank }}=0.0046$.

a function of $T_{b b}$. The green line is the calculated power of Eq. 3 multiplied by an effective number of detected modes $\eta_{\text {eff }}=1.20$ in this instance. Again the pyramidal horns behave in a few-moded fashion for this band, and again the measurement dynamic range exceeds $37 \mathrm{~dB}$.

We found $\eta_{\text {eff }}=1.05 \pm 0.1$ for 4 filled S-band pixels, independent of absorber size. Only $78 \mu \mathrm{m}$ meshed S-band pixels were measured giving $\eta_{\text {eff }}=0.3 \pm 0.2$. For the $\mathrm{S}$-band pixels the reduction in $\eta_{\text {eff }}$ due to meshing was greater than expected, possibly indicative of misalignment of the smaller absorbers with respect to the $68 \mu \mathrm{m}$ horn exit apertures.

\subsection{Measurements of cross-talk and stray light}

In this series of measurements one column of an L-band $4 \times 4$ horn array was blanked by a precisionengineered $\mathrm{Cu}$ blanking plate thus ensuring minimal direct light leakage at the horn entrance aperture. The entrance apertures of the open $3 \times 4$ array can be seen in Fig. 8a.

Figure $8 \mathrm{~b}$ shows measurements of $\Delta P_{J}$ due to optical illumination of the same L-band pixel with open and blanked horn. The red circles and blue squares are the detected optical power in each case. 
The solid green line is the single-mode power scaled by $\eta_{\text {eff }}=1.6$ for the open pixel and the solid light blue line is the same power scaled by $\eta_{\text {blank }}=0.0046$, and it should be noted that the blackbody load was heated to significantly higher temperatures even to register a detection. Also, the detected power at $7<T_{b b}<15 \mathrm{~K}$ is exceptionally-well accounted-for by the L-band spectrum, implying that the measurement is not determined by simple heating of the cold stage or electrical cross-talk. We find the cross-coupling $10 \log \left(\eta_{\text {blank }} / \eta_{\text {eff }}\right)=-25.4 \mathrm{~dB}$ in this case.

The cross-talk measurement would be caused if incident $L$-band power incident through the open horns significantly heated the detector chip directly: the TES is sensitive to temperature changes of its heat bath - in this case the Si chip. The saturation power of the TES measured in Fig. 8b was $P_{\text {sat }}=40 \mathrm{fW}$ and its thermal conductance to the chip calculated from dark measurements, evaluated at $T_{0}=90 \mathrm{mK}$, was $\left.G_{b}\right|_{T_{0}}=0.38 \mathrm{pW} / \mathrm{K}$. The total incident optical power through $N=12$ open horns causing saturation via chip heating would then be $P_{\text {tot }}=N \eta_{\text {eff }} P_{\text {sat }} / \eta_{\text {blank }}$. In a simple two heat-capacity model, with conductances $\left.G_{b}\right|_{T_{0}}$ from the chip to the TES and $G_{s}$ from the chip to the cold stage, the observed saturation would be accounted for by $G_{s}=\left.\eta_{\text {eff }} N_{\text {horns }} G_{b}\right|_{T_{0}} / \eta_{\text {blank }} \simeq$ $2 \mathrm{nW} / \mathrm{K}$. This is more than 3 orders of magnitude lower than our previously-measured thermal conductance to the cold-stage of similar-sized chips at these temperatures with comparable thermal sinking. ${ }^{10}$ The ratio $\eta_{\text {eff }} / \eta_{\text {blank }}=-25.4 \mathrm{~dB}$ determines our estimate of the inter-pixel cross talk and simple thermal modeling would rule-out heating of the chip or other cross-talk with a very high degree of confidence.

In a separate series of measurements, a second blackbody source was mounted outside the test system, directly on the $3 \mathrm{~K}$ stage. This stage can be seen as the lower horizontal plate in Fig. 3 (e). For a typical pixel no change in TES Joule power was observable for $T_{b b}$ up to $26 \mathrm{~K}$ the highest temperature used in the tests. The ratio of the typical pixel noise level $P_{n}$ to the single-mode available optical power $P_{\text {opt }}(26 \mathrm{~K})$ gives an estimate of optical stray light from outside the blackbody assembly. We find $\log \left(P_{n} / P_{\text {opt }}(26 \mathrm{~K})\right) \lesssim-66 \mathrm{~dB}$ showing the high degree of optical isolation achieved in the test system design.

\section{Conclusions}

We have described the fabrication, integration and assembly of L- and S-band pixels using micro machined flat backshorts and profiled pyramidal horns for the SAFARI instrument. Excellent uniformity of assembly was demonstrated for the array and backshorts. The high-degree of uniformity of commercial SoI wafers makes the fabrication and integration of large arrays of backshorts straightforward for future instruments. The pixels were optically characterized using a precision optical test facility comprising variable temperature blackbody load, optical filters and optical aperture. Stray light from outside the optical load and its shielding was below the measurement sensitivity and we estimate more than $-66 \mathrm{~dB}$ rejection. We quantified detected optical power for L- and S-band pixels finding that the tapered pyramidal horns behaved in a few-moded way for both bands as expected. We measured an effective number of modes $\eta_{\text {eff }}=1.95 \pm 0.4$ and $\eta_{\text {eff }}=1.05 \pm 0.1$ for L- and $\mathrm{S}$-bands respectively. For meshed L-band pixels we found $\eta_{\text {eff }}=1.24 \pm 0.05$ where the reduction in $\eta_{\text {eff }}$ can be accounted for by the impedance mismatch arising from the meshing. Crosstalk between pixels was measured to be $-25.4 \mathrm{~dB}$ at L-band.

In future work we will extend the work to the fabrication of large linear arrays of M-band detectors with backing plates, filters and horns in a geometry closely determined by the SAFARI focal 
plane requirements.

\section{References}

1 P. Roelfsema, M. Giard, F. Najarro, K. Wafelbakker, W. Jellema, B. Jackson, B. Sibthorpe, M. Audard, Y. Doi, A. d. Giorgio, M. Griffin, F. Helmich, I. Kemp, F. Kerschbaum, M. Meyer, D. Naylor, T. Onaka, A. Poglitch, L. Spinoglio, F. van der Tak, and B. Vandenbussche, "SAFARI new and improved: extending the capabilities of SPICA's Imaging Spectrometer,' in Space Telescopes and Instrumentation 2014: Optical, Infrared and Millimeter Wave, J. M. Oschmann, Jr., M. Clampin, G. G. Fazio and H. A. MacEwen, Ed., Proc. SPIE 9143, 9143K (2014).

2 T. Nakagawa, H. Shibai, T. Onaka, H. Matsuhara, H. Kaneda, Y. Kawakatsu, P. Roelfsema, and the SPICA team, "The next-generation infrared astronomy mission SPICA under the new framework," in Space Telescopes and Instrumentation 2014: Optical, Infrared and Millimeter Wave, J. M. Oschmann, Jr., M. Clampin, G. G. Fazio and H. A. MacEwen, Ed., Proc. SPIE 9143, 9143I (2014).

3 D. J. Goldie, J. R. Gao, D. M. Glowacka, D. K. Griffin, R. Hijmering, P. Khosropanah, B. D. Jackson, P. D. Mauskopf, D. Morozov, J. A. Murphy, M. Ridder, N. Trappe, C. O’Sullivan, and S. Withington, "Ultra-low-noise Transition Edge Sensors for the SAFARI L-band on SPICA," in Millimeter, Submillimeter, and Far-Infrared Detectors and Instrumentation for Astronomy VI, W. S. Holland and J. Zmuidzinas, Ed., Proc. SPIE 8452, 84520A (2012).

4 M. D. Audley, G. de Lange, M. Ranjan, J. R. Gao, P. Khosropanah, M. L. Ridder, P. D. Mauskopf, D. Morozov, S. Doherty, N. Trappe, and S. Withington, "Measurements of the Optical Performance of Prototype TES Bolometers for SAFARI,” J. Low Temp. Phys. 176, 755-760 (2014).

5 P. Khosropanah, R. Hijmering, M. Ridder, J. R. Gao, D. Morozov, P. D. Mauskopf, N. Trappe, C. O’Sullivan, A. Murphy, D. Griffin, D. Goldie, D. Glowacka, S. Withington, B. D. Jackson, M. D. Audley, and G. de Lange, "TES Arrays for the Short Wavelength Band of the SAFARI Instrument on SPICA," in Millimeter, Submillimeter, and Far-Infrared Detectors and Instrumentation for Astronomy VI, W. S. Holland and J. Zmuidzinas, Ed., Proc. SPIE 8452, 845209 (2012).

6 D. Morozov, R. Sudiwala, P. A. R. Ade, D. J. Goldie, D. M. Glowacka, S. Withington, and N. A. Trappe, "Optical characterization of a camera module developed for ultra-low NEP TES detector arrays at FIR wavelengths," in These Procs., (2016).

7 D. M. Glowacka, M. Crane, D. J. Goldie, and S. Withington, "A fabrication route for arrays of ultra-low-noise transition edge sensors on thin silicon nitride for space applications," J. Low Temp. Phys. 167, 516-521 (2012).

8 Thomas Keating Ltd. http: / /www. terahertz.co.uk/.

9 PolyTech TMS-100. http: //www. polytec.com.uk/.

10 K. Rostem, D. M. Glowacka, D. J. Goldie, and S. Withington, "Thermal conductance measurements for the development of ultra-low noise transition edge sensors with a new method for measuring the noise equivalent power," in Millimeter and Submillimeter Detectors and Instrumentation for Astronomy IV, W. D. Duncan, W. S. Holland, S. Withington, and J. Zmuidzinas, Eds., Proc. SPIE 7020, 70200L (2008). 